EPJ Web of Conferences 103, 03006 (2015)

DOI: 10.1051/epjconf/201510303006

(C) Owned by the authors, published by EDP Sciences, 2015

\title{
Resonant Broadband Memory in Tripod-Type Atomic Ensemble
}

\author{
A.S. Loseva, T.Yu. Golubeva, and Yu.M. Golubev \\ St. Petersburg State University, 198504 St. Petersburg,Stary Petershof, ul. Uljanovskaya, 3, Russia
}

We have considered theoretically a protocol of the broadband quantum memory based on the resonant interaction of fields with atoms in a tripod-type configuration. It is shown that quantum properties of light (particularly, squeezing) can be effectively mapped and then retrieved from the long-lived spin coherences. We discuss two readout configurations: the double-channel simultaneous readout and the single-channel successive one. In the first scheme a single pulse with quantum properties close to the initial signal is formed at the output of the memory cell. In the second case two pulses separated in space and time are formed in the entangled state.

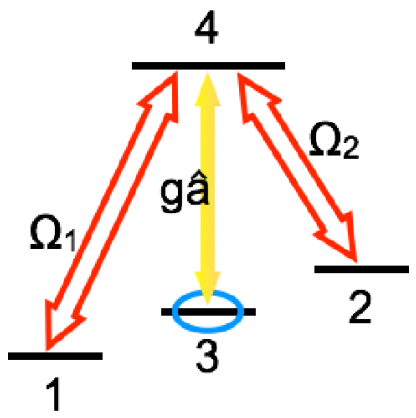

Figure. 1. Tripod-type atomic configuration, $\Omega_{1}$ and $\Omega_{2}$ - Rabi frequencies of control fields, $\hat{a}-$ the amplitude of the signal field.

${ }^{\text {a }}$ Corresponding author: alex.s.losev@gmail.com

This is an Open Access article distributed under the terms of the Creative Commons Attribution License 4.0, which permits unrestricted use, distribution, and reproduction in any medium, provided the original work is properly cited. 